\title{
Observations of Hysteresis Between Flare Index and Some Solar Indices
}

\author{
A. Özgüç and T. Ataç \\ Boğaziçi University, Kandilli Observatory and Earthquake Research \\ Institute, Cengelköy, Istanbul, Turkey
}

\begin{abstract}
Using flare index data sets for solar cycles 21 and 22 we find that flare index and some solar activity indicators show a hysteresis phenomenon. It is observed that total sunspot area, mean magnetic field and coronal index follow different paths for the ascending and descending phases of the solar cycles while saturation effect exists at the extreme phases. However, we notice that the separations between the paths are not the same during the past two cycles of 21 and 22 .
\end{abstract}

\section{Introduction}

Some recent works suggest that certain pair of the solar activity indices show hysteresis, solar cycle dependent differences in their relative variations (Tripathy et al., 2000; Bachman \& White, 1994; Harvey, 1992; Donnelly, 1991). It is showed some of these activity indicators like $10.7 \mathrm{~cm}$ microwave flux, Ca II $\mathrm{K}$ index and $L \alpha$ irradiance at $121.6 \mathrm{~nm}$ follow different paths for the ascending and descending phases of the two solar cycles. Bachman \& White, in particular, express confidence that hysteresis is a real phenomenon, not a result of instrumental effects, and estimate lag times of activity indices compared to sunspot number.

In this study, we expand upon the above works try to show that hysteresis is present between the flare index (FI) and some other activity indices like total sunspot area (TSA), mean magnetic field (MMF) and coronal index (CI) during solar cycles 21 and 22. Although hysteresis shapes amongst several indices have been found in the past (Bachman \& White, 1994), this is the first time that such relations are seen in flare index. Further we search whether hysteresis effect is indeed a feature of solar activity during the past two cycles (21 and 22). However, there is some evidence that this hysteresis is different for the two successive cycles.

\section{Solar Activity Index Data Sets}

FI, it is of value as a measure of the short-lived activity on the Sun. The daily sums of the index for the northern and the southern hemispheres and for the total surface are divided by the total time of observation of that day. Because the time coverage of flare observations is not always complete during a day (sometimes $75 \%$ or $90 \%$ ), it is corrected by dividing by the total time of 
observations of that day to place the daily sum of the flare index on a common 24-hour period (Ataç \& Özgüç, 1998). It is newly calculated by us for the cycles 21 and 22. MMF, Stanford University, Wilcox Solar Observatory's measurement of the net magnetic field intensity in Micro Teslas summed over the disk. Such integrated light measurements of the mean solar magnetic field have been made daily since May 1975 (Scherrer et al., 1977). TSA, these are observed, measured and compiled by Royal Greenwich Observatory and USAF/NOAA. They are taken from the web page of Marshall Space Flight Center. CI, it is derived by Rybansky et al. (1994) from the measurements of the total energy emitted by the Sun's outermost atmoshperic layer (the corona) at a wavelength of 530.3 $\mathrm{nm}$. It gives a radiant energy emitted by the entire visible corona within Fe XIV spectral line. Lomnický S̆tit in Slovak Republic served as the reference station for calculating the index.

Table 1. Results of the correlations between the flare index and the other solar activity indices we used.

\begin{tabular}{llll|lll}
\hline \multicolumn{3}{c|}{ Cycle 21 } & \multicolumn{3}{c}{ Cycle 22 } \\
\hline Index & Asc. p. & Des. p. & Total C. & Asc. p. & Des. p. & Total C. \\
\hline TSA & 0.98 & 0.97 & 0.95 & 0.99 & 0.99 & 0.91 \\
MMF & 0.98 & 0.95 & 0.91 & 0.86 & 0.96 & 0.99 \\
CI & 0.99 & 0.98 & 0.90 & 0.98 & 0.99 & 0.99 \\
\hline \hline
\end{tabular}

\section{Analysis}

In Figure 1, the flare index values are plotted against TSA, MMF and CI for solar cycles 21 and 22 . We choose flare index to be the abscissa of all the plots in this figure, in order to display the effects of hysteresis most clearly. We perform the 365-day running means using only common days of data to produce smoother hysteresis patterns. The plotted curves of cycle 21 (left panel) clearly exhibit a hysteresis. The flare index does not follow the same path in the rising and the falling parts of this cycle; the descending part always follows a higher track than the ascending one. Arrows indicate the direction of time. Line styles of the individual plots are the same in all the plots. The thicker sections of the curves correspond to the rising half of the cycle (e.g., flare index increasing with time). The dashed lines are linear regression fits of all points in each plot. The saturation effects are seen in all the plots at the extreme phases.

We cannot observe the same phenomenon for TSA in cycle 22 (Figure 1, right panel). It does not show a hysteresis; although FI follows different paths for the ascending and the descending phases of cycle 22 but the separation between the two paths are small and within the error limits. The error bars at the top left corner of the right panel indicate $1 \sigma$ values of that plot. MMF and CI show a hysteresis also in this cycle, but contrary the ascending branchs follow a higher track than the descending one. The saturation effects are also seen at the extreme phases in those two plots of the right panel.

We carried out the Spearman's rank correlation between the flare index and the other indices we used. The correlation coefficents for the ascending and the descending branches and for the total cycles of 21 and 22 are summerised in 


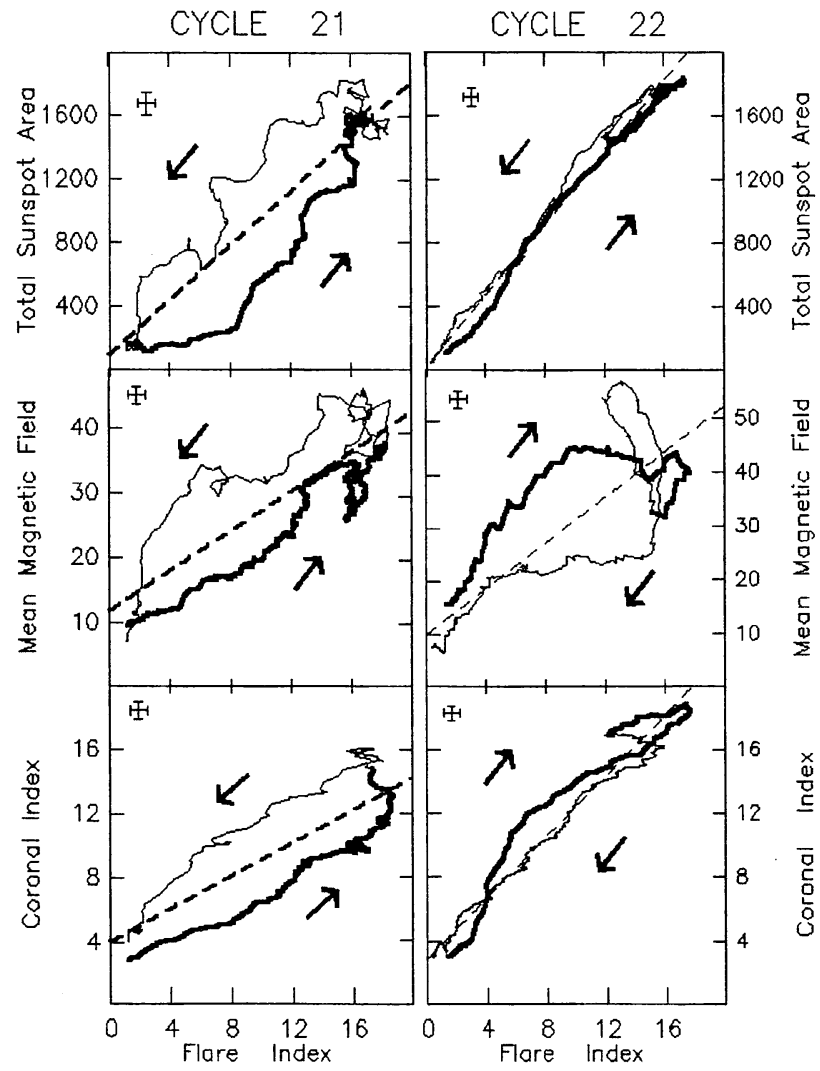

Figure 1. Scatterplots showing the hysteresis phenomenon for total sunspot area, mean magnetic field and coronal index, versus flare index. Solid thin and thick lines represent 365-day running means of the points. The thick lines show ascending branches while the thin lines descending branches of cycles 21 and 22 . The dashed lines are linear regression fits of the points.

Table 1. The correlation coefficents are close to unity; however, one should also bear in mind that the correlations amongst the indices we used here for the same period are also high, varying from 0.80 to 0.98 . The corresponding values of the calculated probabilities of having null correlation are not shown because in all cases they are smaller than $10^{-10}$.

\section{Discussion}

The choise of activity indicator versus flare index may strongly influence the resulting hysteresis in a given cycle. For example for cycle 21, all the indices we used show a hysteresis when they are plotted against FI. However, for cycle 22, we cannot observe a hysteresis on TSA versus FI. Although there is a hysteresis 
between FI and MMF and CI but the direction of the time is opposite when we compare with cycle 21 (e.g., ascending branchs follow higher tracks than the descending ones). Jiménez-Reyes et al. (1998) showed that a hysteresis exists between frequency shifts of odd and even-degree p-modes themselves and also two activity indices (radio flux at $10.7 \mathrm{~cm}$ and the magnetic index from Kitt Peak (Harvey, 1984)) for cycle 22. Comparing the shapes of the hysteresis found, they suggested that the hysteresis shape could be also due to time delayed responses to one single phenomenon which can be located deeper in the Sun. Moreno-Insertis \& Solanki (2000) reported that a hysteresis also exists between low-degree p-mode frequencies and magnetic flux. They suggested that if a given activity indicator basically depends on the magnetic flux distribution at a rnge of latitudes only, then a hysteresis can also appear when plotting the frequency shift of one mode against that activity indicator. Finally Tripathy et al. (2000) found that the intermediate degree frequencies of solar cycle 22 show a hysteresis with the magnetic indices whereas no such effect exists for the radiative indices.

Bachman \& White (1994) estimated lag times for all possible pairs of indices by offsetting one index in time untill the hysteresis curve collapses into roughly a straight line. However we could not estimate lag times for the pairs of indices because of the shape and the width of the hysteresis which vary from cycle to cycle (e.g., for one cycle lag time to be found 40 days and for the next cycle is it found 180 days). To summarise, although hysteresis warrarts serious consideration as a possible long-term feature of solar activity but the shape and width differences between the two cycles are, however, unexpected.

Acknowledgments. This work was supported by Boğaziçi University Research Fund by the project of 00T101.

\section{References}

Ataç, T. \& Özgüç, A. 1998, Solar Phys. 180, 397

Bachman, K. T. \& White, O. R. 1994, Solar Phys. 150, 347

Donnelly, R. F. 1991, J. Geomag. Geoelectr. Suppl. 43, 835

Harvey, K. 1992 in Workshop on the Solar Electromagnetic Radiation Study for Solar Cycle 22, ed. R. F. Donnelly (Boulder: NOAA), 113

Harvey, J. W. 1984, in Proc. Workshop on Solar Variability on Active Region Time Scales, ed. G. Chapman, H. Hudson \& B. Labonte (NASA CP 2130), 197

Jiménez-Reyes, S. J., Régulo, C., Pallé, P. L., \& Roca Cortés, T. 1998, A\&A 329,1119

Moreno-Insertis, F., \& Solanki, S. K. 2000, MNRAS 313, 411

Rybansky, M., Rusin, V., Gaspar, P., \& Altrock, C. R. 1994, Solar Phys. 152, 487

Scherrer, H. P., Wilcox, M.J., Svalgaard, L., Duvall, L. T., Dittmer, H. P., \& Gustafson, E. K. 1977, Solar Phys. 54, 353

Tripathy, S. C., Kumar, B., Jain, K., \& Bhatnagar, A. 2000, J. Astrophys. Astr. in press 